\title{
Dynamic effects on the spin-wave spectrum of the bcc thin film
}

Sławomir Mamica ${ }^{\mathrm{a}}$

Faculty of Physics, Adam Mickiewicz University in Poznań, ul. Umultowska 85, 61-614 Poznań, Poland

Received 25 July 2014 / Received in final form 27 October 2014

Published online 8 December 2014

(c) The Author(s) 2014. This article is published with open access at Springerlink.com

\begin{abstract}
In the present paper we consider a ferromagnetic thin film with exchange interaction between nearest and next-nearest neighbours. Using a microscopic model based on the Heisenberg Hamiltonian we investigate how the propagation parallel to the surface of the film affects the spin-wave spectrum. Due to its wave-vector dependence the effective coupling between lattice planes parallel to the surface can be of ferromagnetic or antiferromagnetic character, or it can vanish completely, depending on the propagation of spin waves. When the effective coupling vanishes, the film separates into subsystems in which spin waves propagate independently. Antiferromagnetic effective coupling for certain wave vectors implies reversed mode order in the spectrum (with the optical mode at the bottom and the acoustic one at the top). Interestingly, this effect occurs also when all the exchange interactions in the considered system are ferromagnetic.
\end{abstract}

\section{Introduction}

The properties of the magnetic thin film underlie a number of effects that occur in systems such as magnetic multilayers of thin-film magnonic crystals [1-5]. Therefore, for more than half a century the magnetic thin film has remained the subject of keen interest, additionally stimulated by the immense progress in the measurement techniques and fabrication technology of such systems [6-12]. A particular class of phenomena is related to in-plane propagation of spin waves, i.e. their propagation parallel to the surface. Propagating surface waves of the DamonEshbach type can exhibit non-reciprocity [13,14], with the frequency versus wave vector dependence different for opposite directions of propagation. In the case of purely exchange spin waves in-plane propagation leads to surface localization in films with a natural surface $[12,15-17]$ and to a collapse of the bulk band, an effect in which all the bulk modes degenerate to a single frequency for certain wave vectors $[18,19]$.

Taking account of both the short-range and longrange interactions results in very interesting effects in a variety of systems, not necessarily magnetic [20-24]. In magnetic systems, the long-range dipolar interaction causes the complete bandgap to open in magnonic crystals [25-28], generates the vortex configuration in magnetic dots [29,30], and is responsible for the splitting of the spectrum into subbands $[31,32]$ and the occurrence of negative group velocity [33] in thin films. In the ferromagnetic thin film in the exchange regime, taking into account the interaction between next-nearest neighbours (NNN) will result in subsurface localization [34,35].

\footnotetext{
a e-mail: mamica@amu.edu.pl
}

In the present paper we focus on the dynamic (propagation) spin-wave effects with NN and NNN exchange interaction taken into account. Neglecting the dipolar interaction works well in systems with sufficiently strong exchange interaction, such as cobalt ultrathin films [12], $\mathrm{Fe} / \mathrm{Pt}$ multilayers [36], europium monochalcogenide magnetic semiconductors [37], or in the case of weakly nonlinear spin waves $[38,39]$. In the planar model used in the present study the film is divided into a number of layers representing lattice planes parallel to the surface, which reduces the problem to that of a one-dimensional finite chain with effective coupling between its elements (lattice planes) [40]. In our microscopic model this effective coupling reflects the total influence of exchange interactions from neighbouring spins taking into account NN and NNN. The effective coupling can be either ferromagnetic $(\mathrm{FM})$, in which case it favours excitations with in-phase precession of the spins in neighbouring planes, or antiferromagnetic (AFM), energetically favouring antiphase precession (if the external field saturates the sample, if not the situation is opposite). By analogy with phonons [41], the former type of excitation is referred to as acoustic, and the latter as optical. In the case of phonons the acoustic mode has a lower energy than the optical mode; the same pattern is considered the normal order of modes in the spin-wave spectrum. If the energy of the optical mode is lower than that of the acoustic mode, the spin-wave spectrum has a reversed mode order. Mode order reversal in the spin-wave spectrum occurs as a consequence of AFM (exchange or dipolar) interaction between neighbouring magnetic layers and as such is widely discussed in the literature [42-48]. Here we demonstrate that in in-plane propagation the mode order can be reversed even if both 

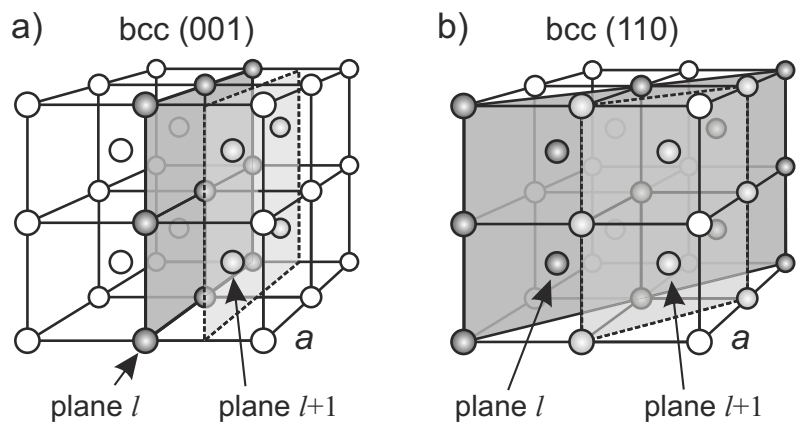

Fig. 1. Neighbouring lattice planes parallel to the surface for (a) bcc(100) and (b) bcc(110) thin film.

the NN and NNN interactions are ferromagnetic. Another in-plane propagation effect studied here is the vanishing of the effective coupling between lattice planes, resulting in the separation of subsystems within the film, with spin waves propagating independently in each subsystem.

The paper is organized as follows. Section 2 presents the main assumptions of the adopted model. Sections 3 and 4 discuss the case of the bcc thin film to analyse the influence of the NNN interaction and the in-plane propagation of spin waves on the effective coupling between sublayers and consequently on the spin-wave spectrum. Special attention is paid to two effects, the mode order reversal and the separation of excitations in independent subsystems. Major results are summarized in Section 5.

\section{Theoretical approach}

For the description of the considered thin film we use the planar model. The sample is assumed to be infinite and homogeneous in the directions parallel to its surface, and finite and in general heterogeneous in the direction perpendicular to the surface. Along the perpendicular direction the film is divided into a number of lattice planes parallel to the surface. In such a system there is a spin in each lattice site; its position is defined by the number $l$ of the plane in which it is situated (Fig. 1), and the vector $j$ indicating its position within this plane. A spin can have neighbors in the same lattice plane $(j \in l)$, in neighboring planes $(\boldsymbol{j} \in l+1)$, as well as in further planes (the NN and NNN distribution in cubic thin films is analyzed in detail in Ref. [49]).

If we assume uniform NN and NNN exchange interaction, the Hamiltonian of the considered system takes the form:

$$
\widehat{\mathcal{H}}=-2 J \sum_{l \boldsymbol{j} ; l^{\prime} \boldsymbol{j}^{\prime}} \widehat{\boldsymbol{S}}_{l \boldsymbol{j}} \cdot \widehat{\boldsymbol{S}}_{l^{\prime} \boldsymbol{j}^{\prime}}-2 J_{N} \sum_{l \boldsymbol{j} ; l^{\prime} \boldsymbol{j}^{\prime}} \widehat{\boldsymbol{S}}_{l \boldsymbol{j}} \cdot \widehat{\boldsymbol{S}}_{l^{\prime} \boldsymbol{j}^{\prime}}
$$

where $J$ and $J_{N}$ are the NN and NNN exchange integrals, respectively; the symbols $l^{\prime} \boldsymbol{j}^{\prime}$ refer to the nearest and nextnearest neighbors, respectively, of the spin $l \boldsymbol{j}$. An external magnetic field, assumed to be strong enough to saturate the film, is applied to the system in the direction perpendicular to the surface. Under this assumption the Zeeman energy, shifting the spin-wave spectrum uniformly, can be omitted in our considerations.

The procedure of diagonalization of the Hamiltonian (1) involves the Holstein-Primakoff transformation (mapping to boson operators), followed by the Fourier transformation in the plane of the film and the Bogolyubov-Tyablikov transformation in the direction perpendicular to it (mapping to the wave-vector space). The procedure is described in detail in many papers and books, see, e.g., $[40,49,50]$, thus here we would like to underline only the consequence of the assumptions the method is based on: it is valid for low concentration of the spin waves, which means low temperatures in the case of thermal magnons, and for samples rather large in the film plane (because of the Fourier expansions in these directions). The final outcome is a band matrix, which for the surface cuts considered here reads:

$$
\widehat{\mathcal{H}}=\left[\begin{array}{ccccccc}
R-a & C & D & & & & \\
C & R-b & C & D & & & \\
D & C & R & C & D & & \\
& \ddots & \ddots & \ddots & \ddots & \ddots & \\
& & D & C & R & C & D \\
& & & D & C & R-c & C \\
& & & & D & C & R-d
\end{array}\right]
$$

where $\widehat{\mathcal{H}}$ is the $L \times L$ matrix, $L$ being the number of layers forming the thin film. Such a matrix describes the behaviour of a chain with the interaction between its elements described by the off-diagonal entries: $C$ refers to the interaction between nearest elements in the chain, and $D$ to the interaction between next-nearest ones. Other entries have following meanings: $R$ represents the on-site energy; $a, d$, and $b, c$ are pairs of surface and subsurface parameters, respectively (see, e.g., [40] for more details). In the problem at hand the role of chain elements is played by lattice planes parallel to the surface; the interaction between them has an effective character and can depend not only on the exchange interaction, but also on the surface cut (NN and NNN distribution) and the in-plane component $\boldsymbol{k}_{\|}$of the wave vector. The diagonal elements of the matrix describe the on-site energy, i.e. the energy of each plane. Note that the Zeeman energy, as an on-site energy, only affects the diagonal entries, shifting the whole spectrum of eigenvalues in a uniform way.

The propagation of spin waves in a thin film is limited to the plane parallel to the surface, which is reflected in the dependence of the elements of the Hamiltonian (2) on the in-plane component of the wave vector. Therefore we shall only consider the wave-vector dependence of the spin-wave spectrum in the surface Brillouin zone (SBZ). For the surface cut bcc(001) the SBZ is a square with a side length of $2 \pi / a$, where $a$ is the lattice constant of the three-dimensional bcc structure. For the surface cut bcc(110) the SBZ has the shape of an irregular hexagon (Fig. 2a), with the wave vector $\boldsymbol{k}_{\|}$described by rather complicated formulas which impacts on the elements of the Hamiltonian. For simplification purposes, following the paper [51], we introduce two variables, $\xi_{1}$ and $\xi_{2}$, assumed to range from -0.5 to 0.5 in the SBZ. 

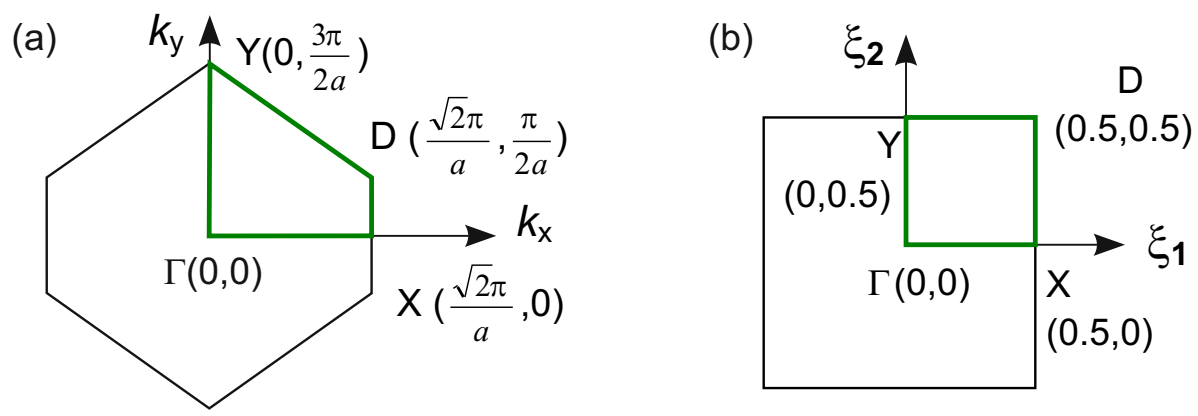

Fig. 2. (a) Surface Brillouin zone and (b) uniform Brillouin zone for the bcc(110) surface cut, with indicated high-symmetry points and their coordinates specified in brackets. The bold green line represents the high-symmetry path along which we have determined the spin-wave spectra presented in Figure 4.

Expressed by these new variables, the SBZ has the shape of a square regardless of its original geometry, and will be henceforth referred to as the uniform zone. The problem of introducing the uniform zone for different surface cuts is discussed in detail in our paper [49]; here we shall only cite the results related to the considered surface cuts.

Figure $2 \mathrm{~b}$ presents the uniform zone, with indicated high-symmetry points, for the bcc(110) surface cut (cf. Fig. 2a). The coordinates $\xi_{1}$ and $\xi_{2}$ of the high-symmetry points are specified in brackets. In this case, the one-toone correspondence that maps each point in the SBZ to a point in the uniform zone is reference [49]:

$$
\boldsymbol{k}_{\|}\left(\xi_{1}, \xi_{2}\right)=\frac{2 \pi}{a}\left[\sqrt{2} \xi_{1}, 2 \xi_{2}\left(\frac{3}{4}-\left|\xi_{1}\right|\right)\right] .
$$

For the bcc(100) surface cut the mapping from the SBZ to the uniform zone amounts to re-scaling the square sides:

$$
\boldsymbol{k}_{\|}\left(\xi_{1}, \xi_{2}\right)=\frac{2 \pi}{a}\left(\xi_{1}, \xi_{2}\right) \text {. }
$$

We shall henceforth use the uniform zone (denoted SBZ) for both considered surface cuts.

\section{Dynamic vanishing of the effective coupling}

In the bcc(001) surface cut NNs lie in the nearest layer $(l+1, \mathrm{NL})$ and NNNs in the next-nearest layer $(l+2$, NNL) [49]. Thus, after reducing the problem to that of a chain, the NN exchange interaction has an influence on the effective coupling between NLs, and the NNN exchange integral on the effective coupling between NNLs. The offdiagonal elements of the Hamiltonian (2) become:

$$
\begin{aligned}
& C=-8 S J \cos \pi \xi_{1} \cos \pi \xi_{2}, \\
& D=-2 S J_{N},
\end{aligned}
$$

where $\xi_{1}$ and $\xi_{2}$ range from -0.5 to 0.5 within the SBZ. Note that the effective coupling $C$ (between first-neighbor planes) depends not only on the NN exchange interaction, but also on the coordinates of the wave vector $\boldsymbol{k}_{\|}$, and consequently changes with spin-wave propagation. By contrast, the effective coupling $D$ (between second-neighbor planes) only depends on the NNN exchange integral.

\section{Case 1: $J_{N}=0$ (collapse of the bulk band)}

If we neglect the NNN exchange interaction, then, according to the formulas (3), the effective coupling between NNLs vanishes; thus, the effective coupling in the considered system is limited to first-neighbour planes. Moreover, for wave vectors at the boundary of the SBZ the coupling $C$ vanishes as well. The matrix (2) becomes a diagonal matrix with eigenvalues $R$ (with the exception of two, which correspond to surface modes). This indicates complete degeneration of all the bulk modes. Illustrating this situation, Figure 3a shows the spin-wave spectrum of a thin film consisting of 11 lattice planes with the NNN exchange interaction switched off. Along the X-M segment (boundary of the SBZ) the off-diagonal element $C$ is zero (green dotted line) and the whole bulk band is degenerate. It should be stressed that the vanishing of the effective coupling is a dynamic effect related to in-plane propagation of spin waves, which for some special wave vectors propagate independently in each lattice plane. (The collapse of the bulk band is discussed in detail in our paper [19].)

\section{Case 2: $\mathrm{J}_{\mathrm{N}}>0$ (separation of subsystems)}

Ferromagnetic NNN exchange interaction implies ferromagnetic effective coupling between NNLs regardless of the spin-wave propagation. By contrast, the effective coupling between NLs does not depend on the NNN exchange, i.e. remains zero at the boundary of the SBZ, as in the case without NNN interaction. Thus, for wave vectors at the boundary of the SBZ only second-neighbour layers are effectively coupled; there is no coupling between firstneighbour layers. In other words, spin waves propagate in two independent subsystems, one consisting of lattice planes with odd numbers and the other of even lattice planes.

Figure $3 \mathrm{~b}$ presents the spin-wave spectrum of a thin film consisting of 11 lattice planes with an NNN exchange integral $J_{N}=0.35 \mathrm{~J}$. Indicative of independent propagation in two subsystems, pairs of nearly degenerate modes occur along the boundary of the SBZ (X-M segment). The independence of excitations in even and odd planes is evident in the profiles at the point $\mathrm{Y}$ in the SBZ, presented in Figure 3d. Besides being surface-localized, the lowest 


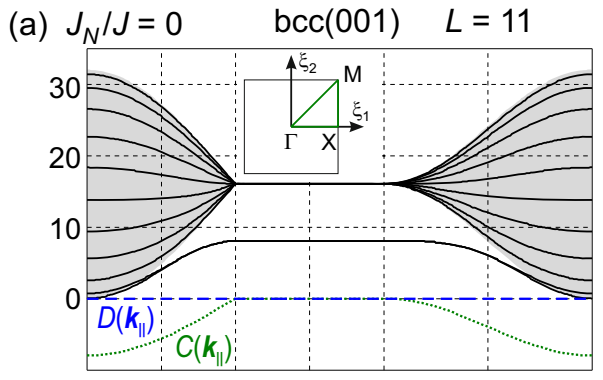

(b) $J_{N} / J=0.35$

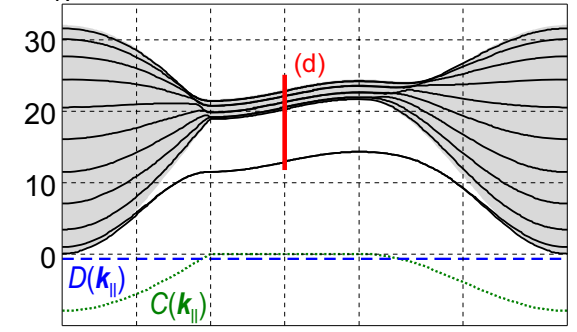

(c) $J_{N} / J=-0.35$

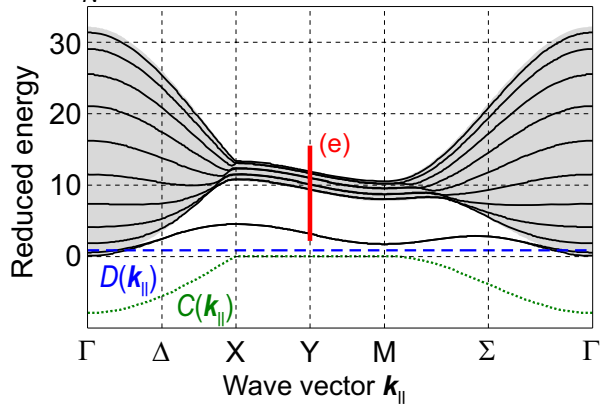

(d) $\quad J_{N} / J=0.35$

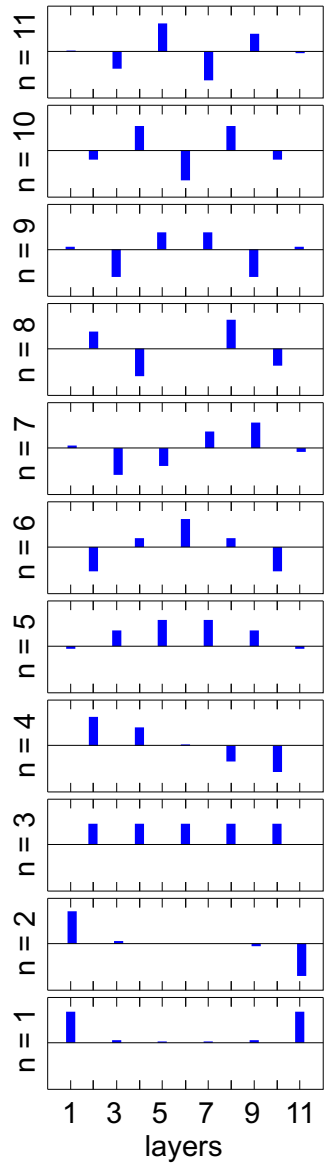

(e) $\quad J_{N} / J=-0.35$

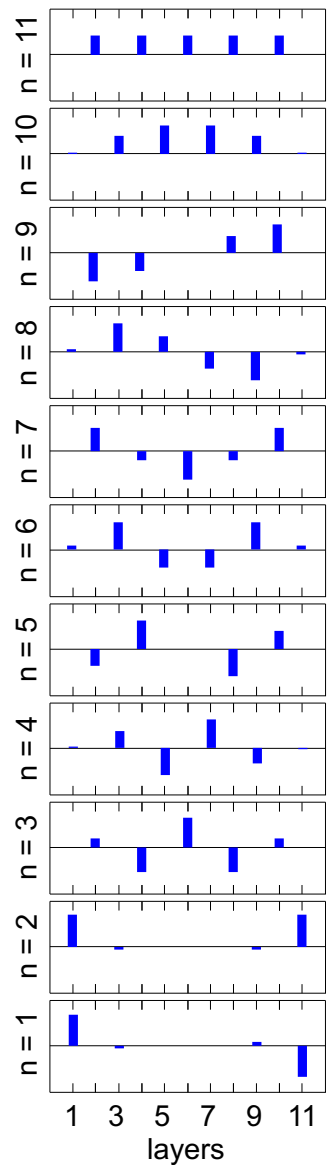

Fig. 3. (a) and (c) Spin-wave spectrum of an 11-ML bcc(001) thin film with three types of NNN exchange interaction: (a) switched off, (b) ferromagnetic, and (c) antiferromagnetic. Solid lines represent the energy of individual modes; the shaded area is the bulk band for in-plane spin waves. Each spectrum is plotted along the high-symmetry path shown in (a), inset. Green dotted line and blue dashed line represent the off-diagonal Hamiltonian matrix elements $C$ and $D$, respectively, plotted versus the in-plane wave vector. Panels (d) and (e) show mode profiles at the point Y of the SBZ for the spectra (b) and (c), respectively.

two modes have a nonzero amplitude only at odd planes. Similarly, the $n=3$ mode is uniform, but only in the subsystem consisting of even planes. Obviously, the separation of these two subsystems within the thin film is a dynamic effect, since it results from the dynamic vanishing of the effective coupling between NLs.

\section{Case 3: $\mathrm{J}_{\mathrm{N}}<0$ (mode order reversal)}

A situation similar to that described above occurs for AFM NNN exchange interaction; however, in this case the effective coupling between NNLs is antiferromagnetic. As we have mentioned above, such coupling favours antiphase precession. Thus, at the boundary of the SBZ, where the effective coupling between NLs vanishes, mode order reversal should be observed along with the separation of subsystems. A sample spectrum for $J_{N}=-0.35 J$ is presented in Figure 3c; Figure 3e shows spin-wave profiles at the boundary of the SBZ. Not only is the lowest mode excited solely in odd planes, but neighbouring amplitudes of precession have opposite signs. A similar pattern is observed in the $n=3$ mode, the lowest mode in the subsystem consisting of even planes. The uniform mode, as in the previous case excited in the subsystem of even planes, now has the highest energy $(n=11)$.

\section{Dynamic mode order reversal}

In the bcc(110) surface cut the most distant NNs and NNNs lie in the neighbouring lattice plane [49]. This means that the off-diagonal elements $D$ of the matrix (2) are zero, and the effective coupling is limited to firstneighbour planes in the considered system. The element $C$, describing the effective coupling between firstneighbour planes, becomes in this case:

$$
C=-4 S\left(J \cos \pi \xi_{2}+J_{N} \cos \left(2 \pi \xi_{1}+\pi \xi_{2}\right)\right) .
$$

If we neglect the NNN exchange interaction $\left(J_{N}=0\right)$, an effect similar to that observed for the bcc(001) surface cut will take place, i.e. the bulk band will collapse 
(a) $\quad \operatorname{bcc}(110) \quad L=15 \quad J_{N} / J=0.35$

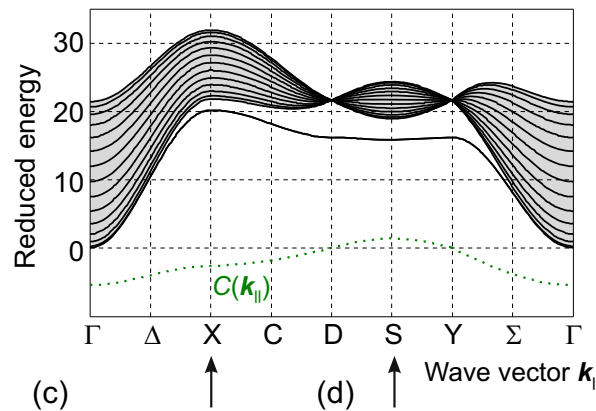

(c)
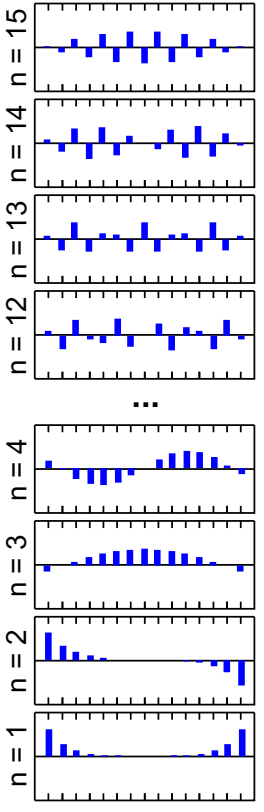

layers
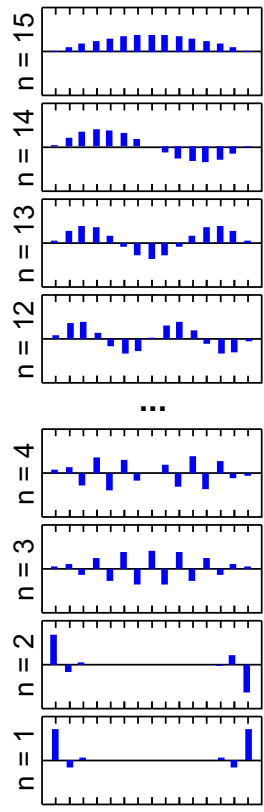

layers (b) $\quad \operatorname{bcc}(110) \quad L=15 \quad J_{N} / J=-0.35$
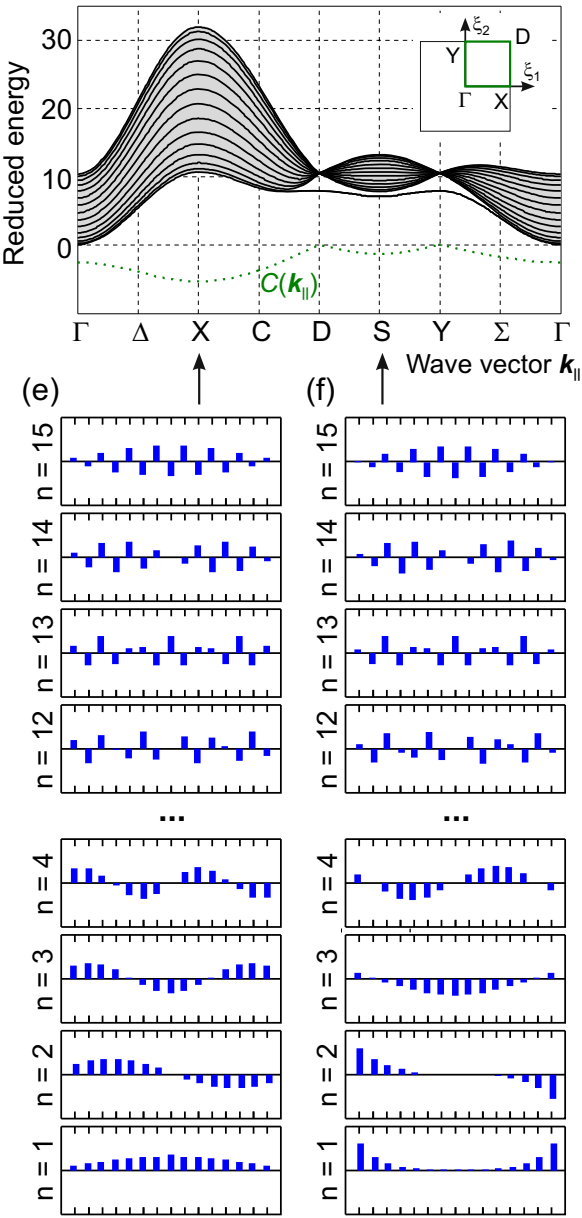

layers
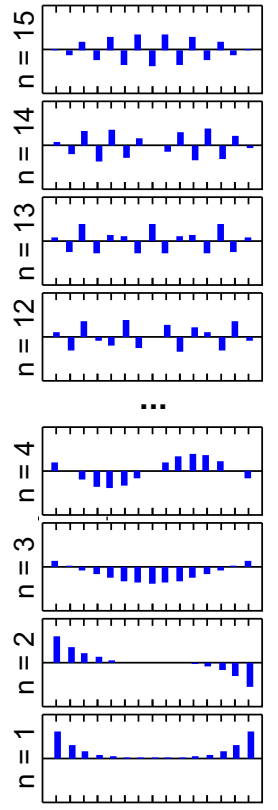

layers

Fig. 4. (a, b) Spin-wave spectrum of a 15-ML thick bcc(110) thin film with (a) ferromagnetic and (b) antiferromagnetic NNN interaction. Solid lines represent the energy of individual modes; the shaded area is the bulk band for in-plane spin waves. Each spectrum is plotted along the high-symmetry path shown in (b), inset. The green dotted line represents the off-diagonal Hamiltonian matrix element $C$ plotted versus the in-plane wave vector. (c) and (f) Profiles of the lowest four and highest four modes in the spectrum at the SBZ points indicated by arrows. Note the reversed mode order for positive values of $C$ : the lowest mode is optical and the highest one acoustic in this case.

at one of the edges of the SBZ (segment D-Y in Fig. 2). For $J_{N}>0$, or ferromagnetic NNN exchange interaction, the band collapses at the high-symmetry points $\mathrm{D}$ and $\mathrm{Y}$; between these points $C>0$ and the order of modes in the spectrum is reversed.

Figure 4a shows a sample spin-wave spectrum obtained for a bcc(110) thin film consisting of $L=15$ lattice planes with $J_{N}=0.35 J$; Figures $4 \mathrm{c}$ and $4 \mathrm{~d}$ present the spinwave profiles determined at points $\mathrm{X}$ and $\mathrm{S}$, respectively, in the SBZ. At the $\mathrm{X}$ point the spin-wave spectrum has a normal order of modes: the lowest mode is acoustic and the highest one optical. By contrast, at the $\mathrm{S}$ point the order of modes is reversed. Interestingly, in contrast to the case of bcc(001), here the reversal takes place for ferromagnetic both NN and NNN exchange interactions. In the case considered the wave vectors that fulfil the condition $C=0$ divide the SBZ into two parts, with normal or reversed order of modes in the spin-wave spectrum. Fig- ure 5 presents this division of the SBZ versus the $J_{n} / J$ ratio. As indicated by the plot, higher NNN exchange integral corresponds to larger region of reversed mode order.

Also for antiferromagnetic NNN exchange interaction the bulk band collapses at the points D and Y in the SBZ, but this time the collapse is not accompanied by mode order reversal between these points. Figure $4 \mathrm{~b}$ shows the corresponding spin-wave spectrum for $J_{N}=-0.35 \mathrm{~J}$; Figures $4 \mathrm{e}$ and $4 \mathrm{f}$ present spin-wave profiles determined at the $\mathrm{X}$ and $\mathrm{S}$ points, respectively, in the SBZ. At both points acoustic modes have the lowest energy and optical modes the highest; thus, though the spectrum resembles that obtained for FM exchange interaction (cf. Fig. 4a), the order of modes is not reversed. This is an obvious consequence of the wave-vector dependence of the off-diagonal element $C$ : for antiferromagnetic NNN exchange interaction its value does not exceed zero for any wave vector in the SBZ. 


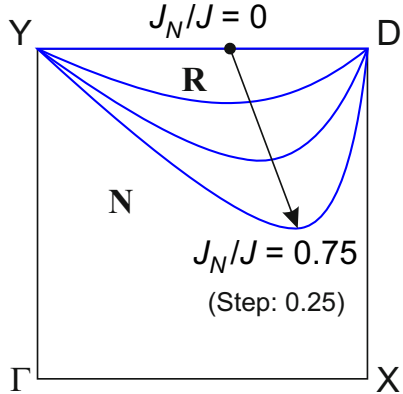

Fig. 5. Two domains in the bcc(110) uniform surface Brillouin zone, with normal $(\mathrm{N})$ or reversed $(\mathrm{R})$ mode order in the spinwave spectrum, for ferromagnetic NNN interaction. The curve representing the boundary between the domains moves in the direction indicated by the arrow with growing $J_{N} / J$. For the limit value $J_{N} / J=0$ the reversal domain vanishes (its boundary amounts to the D-Y segment).

\section{Conclusions}

We have analysed the effects related to in-plane propagation of spin waves in a ferromagnetic thin film. By treating the film as a system of lattice planes parallel to the surface the problem is reduced to that of a one-dimensional chain with effective interlayer coupling dependent on the model parameters, such as the exchange integral or the surface cut. The coupling depends also on the wave vector, which implies its sensitivity to the propagation of spin waves. In a special case it can vanish for certain wave vectors, which means that spin waves with these wave vectors will propagate independently in each lattice plane. The band of bulk modes will collapse to a single energy level in this case.

Also, the effective interlayer coupling has a major influence on the order of modes in the spin-wave spectrum. If the effective coupling is ferromagnetic, the acoustic mode, with in-phase precession of spins in neighbouring planes, is the lowest in the spectrum, and the optical mode, with antiphase precession in neighbouring planes, is the highest. When the effective coupling is antiferromagnetic the order of modes is reversed (under the condition that the external field saturates the sample, in other case the neighbouring magnetizations are anti-parallel and the lowest mode is acoustic [52]). In this study we show that AFM interlayer coupling can occur as a propagation effect even if all the exchange interactions in the system are ferromagnetic. The related mode order reversal in the spin-wave spectrum should be observable in BLS (Brillouin light scattering) and SPEELS (spin-polarized electron energy loss spectroscopy) experiments as a collapse, followed by a spectrum with a reversed peak intensity pattern with changing wave-vector length.

Another effect related to in-plane propagation is the separation of two subsystems within the thin film, with spin waves propagating independently in each subsystem. This effect occurs in the case of the bcc(001) surface cut for wave vectors at the boundary of the Brillouin zone. The effective coupling between first-neighbour planes vanishes dynamically, whereas the coupling between second- neighbour planes, being independent of the wave vector, remains nonzero. This results in independent propagation of spin waves in two subsystems consisting of either even or odd lattice planes.

This study has received financial support from the European Community's 7th Framework Programme (FP7/2007-2013), Grant Agreement No. 247556 (NoWaPhen). The author also acknowledges the support from the National Science Centre (NCN) of Poland, Project DEC-2012/07/E/ST3/00538.

\section{References}

1. J. Barnaś, J. Magn. Magn. Mater. 128, 171 (1993)

2. W.P. Zhou, G.H. Yun, Surf. Sci. 553, 75 (2004)

3. S. Neusser, H.G. Bauer, G. Duerr, R. Huber, S. Mamica, G. Woltersdorf, M. Krawczyk, C.H. Back, D. Grundler, Phys. Rev. B 84, 184411 (2011)

4. M.L. Sokolovskyy, J.W. Kłos, S. Mamica, M. Krawczyk, J. Appl. Phys. 111, 07C515 (2012)

5. M. Krawczyk, D. Grundler, J. Phys.: Condens. Matter 26, $123202(2014)$

6. A.R. Ferchmin, Phys. Lett. 1, 281 (1962)

7. P.E. Wigen, C.F. Kooi, M.R. Shanabarger, T.D. Rossing, Phys. Rev. Lett. 9, 206 (1962)

8. P. Grünberg, J. Barnaś, F. Saurenbach, J.A. Fuss, A. Wolf, M. Vohl, J. Magn. Magn. Mater. 93, 58 (1991)

9. I. Zasada, B. Busiakiewicz, L. Wojtczak, J. Magn. Magn. Mater. 312, 58 (2007)

10. C.A.F. Vaz, J.A.C. Bland, G. Lauhoff, Rep. Prog. Phys. 71, 056501 (2008)

11. J. Dubowik, K. Zaleski, H. Glowinski, I. Goscianska, Phys. Rev. B 84, 184438 (2011)

12. R. Vollmer, M. Etzkorn, P.S. Anil Kumar, H. Ibach, J. Kirschner, Phys. Rev. Lett. 91, 147201 (2003)

13. M. Mruczkiewicz, M. Krawczyk, G. Gubbiotti, S. Tacchi, Y.A. Filimonov, D.V. Kalyabin, I.V. Lisenkov, S.A. Nikitov, New J. Phys. 15, 113023 (2013)

14. M. Mruczkiewicz, M. Krawczyk, J. Appl. Phys. 115, 113909 (2014)

15. H. Puszkarski, J.-C.S. Lévy, S. Mamica, Phys. Lett. A 246, 347 (1998)

16. S. Mamica, R. Jozefowicz, H. Puszkarski, Acta Phys. Pol. A 94, 79 (1998)

17. S. Mamica, H. Puszkarski, Mater. Sci. Poland 26, 989 (2008)

18. J.-C.S. Lévy, E. Ilisca, J.L. Motchane, Phys. Rev. B 5, 1099 (1972)

19. S. Mamica, J. Magn. Magn. Mater. 322, 2994 (2010)

20. J. Barnaś, B. Hillebrands, Phys. Stat. Sol. B 176, 465 (1993)

21. C. Dubus, K. Sekimoto, J.-B. Fournier, Proc. R. Soc. A 462, 2695 (2006)

22. A.P. Grosvenor, R.G. Cavell, A. Mar, J. Solid State Chem. 180, $2702(2007)$

23. J.W. Kłos, I.V. Zozoulenko, Phys. Rev. B 82, 081414 (2010)

24. S. Mamica, J.-C.S. Lévy, M. Krawczyk, J. Phys. D 47, 015003 (2014)

25. M. Krawczyk, H. Puszkarski, J.-C.S. Lévy, S. Mamica, D. Mercier, J. Magn. Magn. Mater. 246, 93 (2002) 
26. S. Mamica, M. Krawczyk, J.W. Kłos, Adv. Cond. Mat. Phys. 2012, 161387 (2012)

27. J.W. Kłos, M.L. Sokolovskyy, S. Mamica, M. Krawczyk, J. Appl. Phys. 111, 123910 (2012)

28. S. Mamica, M. Krawczyk, M.L. Sokolovskyy, J. RomeroVivas, Phys. Rev. B 86, 144402 (2012)

29. S. Mamica, J.-C.S. Lévy, Ph. Depondt, M. Krawczyk, J. Nanopart. Res. 13, 6075 (2011)

30. Ph. Depondt, J.-C.S. Lévy, S. Mamica, J. Phys.: Condens. Matter 25, 466001 (2013)

31. M. Krawczyk, S. Mamica, J.W. Kłos, J. Romero-Vivas, M. Mruczkiewicz, A. Barman, J. Appl. Phys. 109, 113903 (2011)

32. S. Pal, B. Rana, S. Saha, R. Mandal, O. Hellwig, J. Romero-Vivas, S. Mamica, J.W. Kłos, M. Mruczkiewicz, M.L. Sokolovskyy, M. Krawczyk, A. Barman, J. Appl. Phys. 111, 07C507 (2012)

33. M. Krawczyk, S. Mamica, M. Mruczkiewicz, J.W. Kłos, S. Tacchi, M. Madami, G. Gubbiotti, G. Duerr, D. Grundler, J. Phys. D 46, 495003 (2013)

34. S. Mamica, H. Puszkarski, J.-C.S. Lévy, Phys. Stat. Sol. B 218, 561 (2000)

35. S. Mamica, H. Puszkarski, J.-C.S. Lévy, Acta Phys. Pol. A 97, 483 (2000)

36. R. Elkabil, K. Benkirane, H. Lassri, A. Hamdoun, M. Abid, A. Berrada, R. Krishnan, Physica A 358, 142 (2005)

37. W. Söllinger, W. Heiss, R.T. Lechner, K. Rumpf, P. Granitzer, H. Krenn, G. Springholz, Phys. Rev. B 81, $155213(2010)$

38. S.A. Nikitov, R.F. Wallis, Phys. Rev. B 50, 998 (1994)

39. Y. Xu, G. Su, D. Xue, Z. Chen, F.-S. Li, Phys. Scr. 64, 92 (2001)

40. H. Puszkarski, Progr. Surf. Sci. 9, 191 (1979)
41. C. Kittel, Introduction to Solid State Physics (Wiley, 2004)

42. P. Grünberg, J. Appl. Phys. 51, 4338 (1980)

43. P. Grünberg, J. Appl. Phys. 52, 6824 (1981)

44. S.M. Rezende, C. Chesman, M.A. Lucena, A. Azevedo, F.M. de Aguiar, S.S.P. Parkin, J. Appl. Phys. 84, 958 (1998)

45. S. Mamica, H. Puszkarski, Acta Physicae Superficierum 5, 5 (2003)

46. R.W. Wang, D.L. Mills, Phys. Rev. B 50, 3931 (1994)

47. R. Zivieri, L. Giovannini, F. Nizzoli, Phys. Rev. B 62, 14950 (2000)

48. R. Zivieri, Solid State Phys. 63, 151 (2012)

49. S. Mamica, H. Puszkarski, in The Effect of Crystallographic Surface Cut on Surface State Existence Conditions in Cubic Thin Films. Application to Magnetic Films in Surface Magnetism and Nanostructures, edited by A. Ghazali, J.-C.S. Lévy (Research Signpost, Kerala, India, 2006), pp. 129-173

50. A.G. Gurevich, G.A. Melkov, Magnetization Oscillations and Waves (CRC Press, 1996)

51. M. Mostoller, A.K. Rajagopal, Phys. Rev. B 25, 6168 (1982)

52. M. Grimsditch, S. Kumar, E.E. Fullerton, Phys. Rev. B $\mathbf{5 4}, 3385$ (1996)

Open Access This is an open access article distributed under the terms of the Creative Commons Attribution License (http://creativecommons.org/licenses/by/4.0), which permits unrestricted use, distribution, and reproduction in any medium, provided the original work is properly cited. 